\title{
Determinación de la resistencia del hormigón en viguetas fabricadas con cemento aluminoso
}

\author{
ANTONIO DE LA FUENTE SÁNCHEZ \\ Ledo. en Ciencias Químicas \\ Laboratorio de Barcelona. Direcció General d'Arquitectura i Habitatge \\ GENERALITAT DE CATALUNYA \\ ESPAÑA
}

Fecha de recepción: 5-III-93

\section{INTRODUCCIÓN}

La resistencia de los hormigones en viguetas de cemento aluminoso es uno de los parámetros más solicitados a los laboratorios de control. La dificultad de su determinación estriba en la gran cantidad de armaduras que suelen llevar las alas de la vigueta, siendo el alma la única zona de la que pueden extraerse muestras de hormigón. Dado que el grueso habitual del alma de la vigueta oscila entre $2 \mathrm{~cm}$ y $4 \mathrm{~cm}$, no se puede hacer un uso fiable de los métodos no destructivos habituales para determinar la resistencia de dicho hormigón.

Por otra parte es difícil determinar los diferentes parámetros que caracterizan a estos hormigones, como pueden ser dosificación, porosidad, etc., ya que la cantidad de muestra necesaria para realizar todos los ensayos es lo suficientemente grande para que su extracción pueda significar un daño importante a la capacidad resistente de la vigueta.

Sabido es que el valor de la resistencia a compresión de un hormigón se ve fuertemente influido por la forma y tamaño de las probetas y tamaño máximo del árido. El diámetro de las probetas ha de ser, al menos, tres veces superior al máximo del árido, según la Norma UNE 83605.

El objeto de este trabajo es correlacionar los valores obtenidos de la resistencia a compresión de microprobetas cilíndricas de $2 \mathrm{~cm}$ de diámetro por 2 ó $4 \mathrm{~cm}$ de altura con aquéllos exhibidos en las probetas normalizadas de $15 \times 30 \mathrm{~cm}$, todas ellas de un hormigón de igual naturaleza. Este trabajo forma parte de una investigación en curso, cuyo fin sería proponer un método de medida y determinación de resistencia a compresión del hormigón en viguetas, ya sean éstas de cemento aluminoso o portland, a través del ensayo de microprobetas extraídas del alma de la vigueta.

En el Laboratorio de Barcelona se han recibido para el ensayo de resistencia a flexión una cantidad de aproximadamente 30 viguetas de hormigón de cemento aluminoso.

En las viguetas, además de los datos habitualmente solicitados (carga de rotura, carga de fisuración y resistencia a tracción de las armaduras), se ha determinado también densidad, porosidad, resistencia del hormigón, contenido en cemento, relación 
agua/cemento $(a / c)$ y el análisis mineralógico por difracción de rayos $X(D R X)$ de la pasta de cemento enriquecida.

\section{MÉTODO EXPERIMENTAL}

Dada la dificultad de estimar la resistencia del hormigón en las viguetas fabricadas con cemento aluminoso, se estudió realizar esta estimación a partir de la extracción de microprobetas del alma de la vigueta

Para comprobar la validez de este método se prepararon o extrajeron las siguientes probetas:

a) Preparación de probetas (con hormigón de cemento portland) normalizadas de $15 \times 30 \mathrm{~cm}$. El cemento utilizado fue un Tipo 1-42,5R (según designación de la prenormativa europea). En todos los casos el tamaño máximo del árido era de $20 \mathrm{~mm}$ y de naturaleza caliza.

b) Preparación de microprobetas cúbicas del mismo hormigon descrito, cuyos lados variaban entre $2 \mathrm{~cm}$ y $3,5 \mathrm{~cm}$.

c) Extracción de microprobetas cilíndricas de $2 \mathrm{~cm}$ de diámetro y $2 \mathrm{~cm}$ ó $4 \mathrm{~cm}$ de altura. Estas probetas cilíndricas se extraen de las probetas normalizadas de $15 \times 30 \mathrm{~cm}$, con una sonda de $2 \mathrm{~cm}$ de diámetro; posteriormente se cortan con una sierra de preparación de muestras para microscopía. Si bien las caras se obtienen suficientemente lisas y paralelas, en el momento de la rotura y a modo de refrentado, se colocan dos cartoncitos entre los platos de la prensa y las caras de las probetas.

De cada probeta de $15 \times 30 \mathrm{~cm}$ se extrajeron entre 6 y 10 micropobetas cilíndricas de $2 \times 4$ centímetros y otras tantas de $2 \times 2 \mathrm{~cm}$.

d) Extracción de microprobetas cilíndricas a partir de viguetas de cemento aluminoso, de $2 \mathrm{~cm}$ de diámetro.

Todas las muestras indicadas en los apartados a, b, cy d fueron ensayadas a compresión. Se observó que cuando las microprobetas se rompian en húmedo sufrían una disminución de resistencias próxima al $20 \%$ del valor de la rotura en seco. Por ello, se rompieron a las 24 horas de su extracción, dejándolas durante este tiempo a humedad y a temperatura ambiente.

Se realizaron, asimismo, ensayos para determinar la porosidad accesible al agua, la dosificación en cemento, así como el contenido en agua sobre los hormigones de cemento aluminoso.

\section{RESULTADOS Y DISCUSIÓN}

La relación encontrada entre la resistencia de las microprobetas cúbicas y las cilíndricas normalizadas de $15 \times 30 \mathrm{~cm}$ fue muy alta (del orden de 1,4). Teniendo en cuenta este resultado, así como que la dificultad de tallado de las probetas sería considerable y el sistema no podría ser utilizado nada más que en el caso de disponer de viguetas en el laboratorio, se desechó la geometría cúbica y se prosiguió el estudio sobre las microprobetas cilíndricas.

Los datos obtenidos sobre probetas de hormigón de cemento portland se dan en la Tabla I.

En la figura 1 se representan las curvas obtenidas a partir de estos valores.

El coeficiente de correlación obtenido entre las probetas de $15 \times 30 \mathrm{~cm}$ y las de $2 \times 4 \mathrm{~cm}$ es de 0,911 , y el obtenido entre las probetas de $15 x$ $x 30 \mathrm{~cm}$ y las de $2 \times 2 \mathrm{~cm}$ es de 0,8874 ; mientras que el coeficiente de variación de la relación $\mathrm{R} 15 \mathrm{x}$ x 30/R2 x 4 es de 5,90\% en las microprobetas de $2 \times 4 \mathrm{~cm}$ y el de la $\mathrm{R} 15 \times 30 / R 2 \times 4$ es de $6,81 \%$ en las de $2 \times 2 \mathrm{~cm}$.

En la Tabla II se muestran los resultados obtenidos sobre las microprobetas extraídas de viguetas de hormigón de cemento aluminoso.

Se comprueba que los coeficientes de variación que presentan la medida de las resistencias hechas sobre las microprobetas extraídas de viguetas de hormigón aluminoso son similares a los obtenidos sobre las microprobetas extraídas de las probetas normalizadas de hormigón de cemento portland de $15 \times 30 \mathrm{~cm}$. Por ello se considera como método útil y preciso para evaluar la resistencia de las viguetas de hormigón de cemento aluminoso.

En el Laboratorio de Barcelona se ha diseñado un sistema que, en breve, será operativo y permitirá extraer microprobetas de las viguetas in situ.

En cuanto a la correlación de los datos de resistencia de las microprobetas con los demas parámetros procedentes del análisis del hormigón de las viguetas, se pueden adelantar las siguientes consideraciones:

- La DRX, si bien aporta datos fundamentales en cuanto al estado y posible evolución del cemento aluminoso, no parece que de los difractogramas pueda extraerse ningún dato que permita evaluar la resistencia del hormigón. Ello, posiblemente, es debido a que en los hormigones elaborados en Cataluña se ha utilizado siempre árido calizo y, por tanto, no se puede diferenciar entre el aporte de caliza del árido y el producido por la carbonatación del cemento. 
TABLA I

\begin{tabular}{|c|c|c|c|c|c|c|}
\hline R $15 \times 30$ & Mic. $2 \times 4$ & $R 15 \times 30 / R 2 \times 4$ & C.V. $2 \times 4$ & MIC. $2 \times 2$ & $R 15 \times 30 / R 2 \times 2$ & C.V. $2 \times 2$ \\
\hline $\begin{array}{l}187 \\
199 \\
204 \\
209 \\
212 \\
215 \\
218 \\
218 \\
223 \\
228 \\
240 \\
240 \\
243 \\
243 \\
252 \\
260 \\
264 \\
266 \\
274 \\
274 \\
275 \\
275 \\
280 \\
283 \\
287 \\
315 \\
343\end{array}$ & $\begin{array}{l}213 \\
199 \\
242 \\
236 \\
234 \\
242 \\
259 \\
237 \\
269 \\
256 \\
268 \\
276 \\
269 \\
286 \\
303 \\
320 \\
282 \\
293 \\
313 \\
320 \\
345 \\
314 \\
314 \\
292 \\
303 \\
315 \\
360\end{array}$ & $\begin{array}{l}0,88 \\
1,00 \\
0,84 \\
0,89 \\
0,91 \\
0,89 \\
0,84 \\
0,92 \\
0,83 \\
0,89 \\
0,90 \\
0,87 \\
0,90 \\
0,85 \\
0,83 \\
0,81 \\
0,94 \\
0,91 \\
0,88 \\
0,86 \\
0,80 \\
0,88 \\
0,89 \\
0,97 \\
0,95 \\
1,00 \\
0,95\end{array}$ & $\begin{array}{r}6,58 \\
7,08 \\
10,75 \\
9,47 \\
9,04 \\
13,18 \\
7,25 \\
9,87 \\
14,25 \\
6,33 \\
10,17 \\
7,75 \\
1,89 \\
10,99 \\
10,00 \\
10,17 \\
12,51 \\
6,14 \\
7,69 \\
15,30 \\
17,11 \\
9,40 \\
7,60 \\
6,11 \\
9,87 \\
9,20 \\
13,40\end{array}$ & $\begin{array}{l}227 \\
250 \\
255 \\
255 \\
272 \\
287 \\
268 \\
245 \\
322 \\
279 \\
277 \\
322 \\
- \\
325 \\
305 \\
- \\
356 \\
322 \\
320 \\
381 \\
359 \\
339 \\
343 \\
326 \\
340 \\
342 \\
405\end{array}$ & $\begin{array}{l}0,82 \\
0,80 \\
0,80 \\
0,82 \\
0,78 \\
0,75 \\
0,81 \\
0,89 \\
0,69 \\
0,82 \\
0,87 \\
0,75 \\
-\overline{0} \\
0,75 \\
0,83 \\
-\overline{0} \\
0,74 \\
0,83 \\
0,86 \\
0,72 \\
0,77 \\
0,81 \\
0,82 \\
0,87 \\
0,84 \\
0,92 \\
0,85\end{array}$ & $\begin{array}{r}11,26 \\
13,37 \\
12,58 \\
15,32 \\
11,90 \\
15,68 \\
8,51 \\
11,95 \\
7,72 \\
9,03 \\
8,80 \\
5,97 \\
- \\
12,30 \\
5,12 \\
- \\
11,11 \\
8,62 \\
7,84 \\
10,99 \\
12,06 \\
7,77 \\
8,10 \\
8,84 \\
7,38 \\
13,76 \\
4,40\end{array}$ \\
\hline \multicolumn{2}{|l|}{ MEDIA } & 0,89 & \multicolumn{2}{|l|}{9,59} & 0,81 & 10,01 \\
\hline \multicolumn{3}{|l|}{ C. VAR. } & \multicolumn{3}{|l|}{$5,90 \%$} & $6,81 \%$ \\
\hline
\end{tabular}

Los valores de la resistencia a compresión se expresan en $\mathrm{kp} / \mathrm{cm}^{2}$.

VIGUETAS

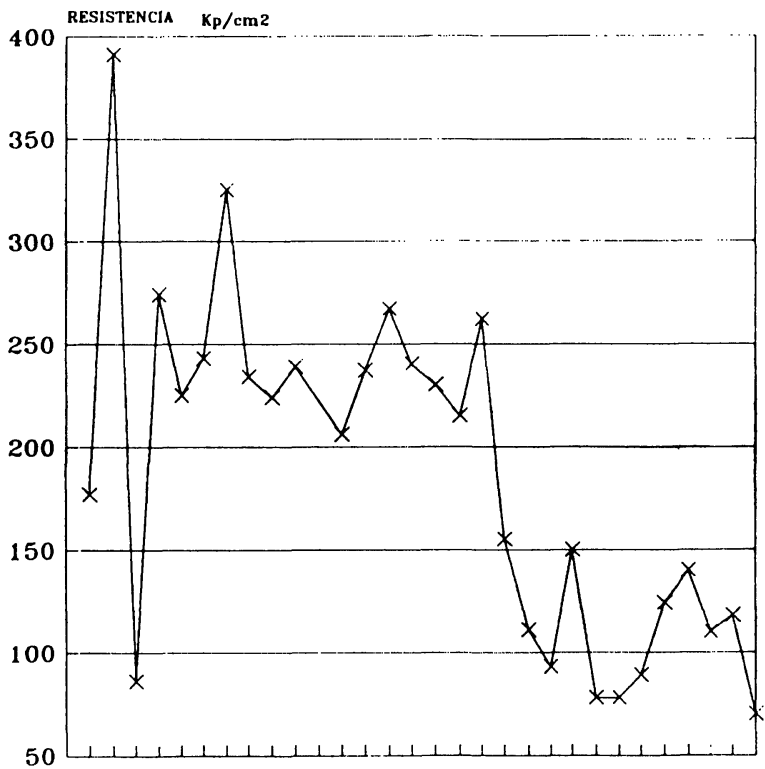

111111111111111111111111111122 122222222223333556677888999902

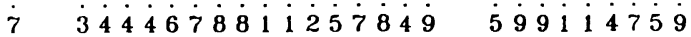
POROSIDAD \%

Fig. 1
MICROPROBETAS

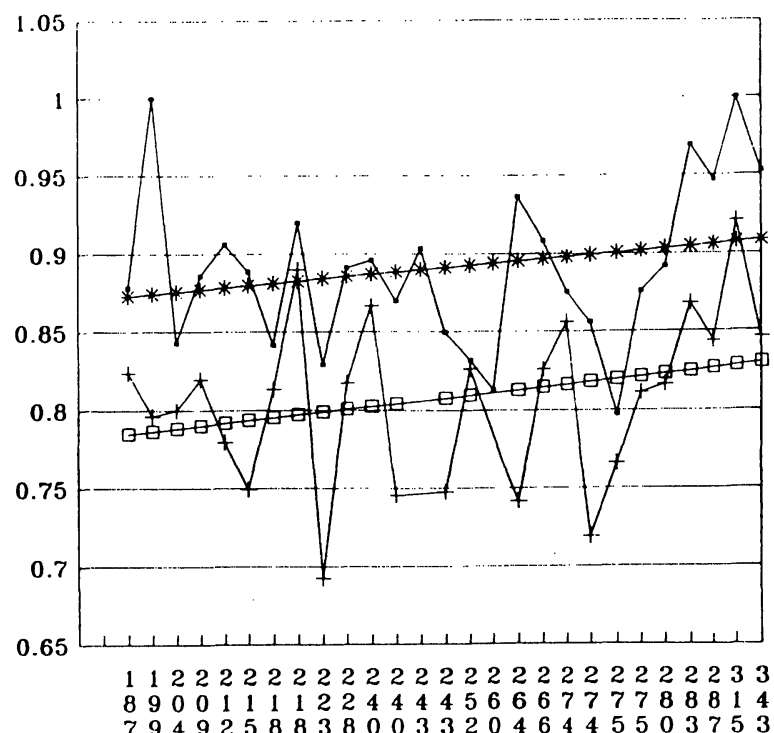

Res. probetas de $15 \cdot 30(\mathrm{Kp} / \mathrm{cm} 2)$

$\rightarrow \mathrm{R} 15 * 30 / \mathrm{R} 2 *$

$+\mathrm{R} 15^{*} 30 / \mathrm{R} 2 * 2$

* $\operatorname{corr} .2 * 4$

Fig. 2 
- Parece posible una correlación entre la relación agua/cemento y la resistencia del hormigón de cemento aluminoso. Con los datos obtenidos hasta el momento (en los cuales la relación a/c varía entre 0,56 y 1,07 ), en ningún caso, con relaciones $\mathrm{a} / \mathrm{c}>0,81$, se han obtenido resistencias superiores a $200 \mathrm{kp} / \mathrm{cm}^{2}$. (Ver tabla II).

- En la figura 2 se representa los valores de la resistencia a compresión frente a las porosidades exhibidas por el hormigón de las viguetas de cemento aluminoso. De dicha gráfica se deduce que existe una porosidad crítica próxima al $16,5 \%$, a partir de la cual ningún hormigón alcanza una resistencia superior a los 155 $\mathrm{kp} / \mathrm{cm}^{2}$.
Como aclaración es preciso decir que los dos hormigones, que en el gráfico presentan una baja porosidad y una baja resistencia, corresponden a los hormigones de más bajo contenido en cemento de todos los estudiados.

- Como apunte de conclusión, mencionar que en todos los casos estudiados se ha observado que, para que un hormigón de cemento aluminoso tenga una resistencia igual o superior a los $200 \mathrm{kp} / \mathrm{cm}^{2}$, han de cumplirse las siguientes condiciones:

1) que su porosidad sea inferior a $16,5 \%$ y

2) que su relación a/c sea inferior a 0,8 .

TABLA II

Resultados sobre microprobetas de hormigón de cemento aluminoso

\begin{tabular}{|c|c|c|c|}
\hline $\begin{array}{c}\text { Porosidad } \\
\%\end{array}$ & $\begin{array}{c}\text { Relación } \\
\text { a/c }\end{array}$ & $\begin{array}{l}\text { Resistencia } \\
\text { microprobetas } \\
\mathrm{kp} / \mathrm{cm}^{2}\end{array}$ & $\begin{array}{c}\text { Coeficiente variación } \\
\text { microprobetas } \\
\%\end{array}$ \\
\hline 11,7 & 1,00 & 177 & 23,16 \\
\hline 12,0 & - & 391 & 11,34 \\
\hline 12,0 & 0,90 & 86 & - \\
\hline 12,3 & 0,71 & 274 & 5,42 \\
\hline 12,4 & 0,56 & 225 & 14,90 \\
\hline 12,4 & 0,58 & 243 & 13,60 \\
\hline 12,4 & 0,74 & 325 & 11,27 \\
\hline 12,6 & 0,62 & 234 & 10,95 \\
\hline 12,7 & - & 224 & 18,09 \\
\hline 12,8 & - & 239 & - \\
\hline 13,1 & - & 206 & - \\
\hline 13,1 & 0,69 & 237 & 7,30 \\
\hline 13,5 & 0,78 & 240 & 12,19 \\
\hline 15,7 & 0,81 & 230 & 13,80 \\
\hline 15,8 & - & 215 & - \\
\hline 16,4 & 0,76 & 262 & 10,14 \\
\hline 16,9 & 0,89 & 155 & 30,24 \\
\hline 17,0 & 0,86 & 111 & - \\
\hline 17,0 & 0,71 & 93 & 10,17 \\
\hline 18,5 & 0,69 & 150 & 5,87 \\
\hline 18,9 & 0,95 & 78 & - \\
\hline 18,9 & 0,95 & 78 & 10,97 \\
\hline 19,1 & 1,07 & 89 & 10,13 \\
\hline 19,1 & 0,75 & 124 & 7,12 \\
\hline 19,4 & 0,90 & 140 & 12,72 \\
\hline 20,5 & 0,89 & 118 & 5,25 \\
\hline 22,9 & 1,00 & 70 & 16,20 \\
\hline
\end{tabular}




\section{A N EXO}

Los métodos utilizados en la realización de los ensayos son los siguientes:

\section{POROSIDAD:}

Dado que por DRX se comprobó la ausencia de aluminatos hexagonales en las muestras, el ensayo se realizó como sigue:

Una muestra de hormigón se mantiene durante 24 horas sumergida en agua en un recipiente cerrado y al vacío, bajo una presión de aproximadamente $1 \mathrm{~mm}$ de $\mathrm{Hg}$; la muestra se pesa seca a $100^{\circ} \mathrm{C}$, sumergida en agua y saturada; la porosidad en $\%$ en volumen es igual a:

$$
\left(P_{\text {saturado }}-P_{\text {seco }} / P_{\text {saturado }}-P_{\text {sumergido }}\right) \cdot 100
$$

La densidad es igual a:

$$
P_{\text {seco }} / P_{\text {saturado }}-P_{\text {sumergido }}
$$

\section{DOSIFICACIÓN EN CEMENTO}

El contenido en cemento del hormigón se realizó mediante la determinación, por absorción atómica, del contenido en alúmina del hormigón; no se tiene en cuenta el posible aporte de alúmina por parte de los áridos, ya que después de varios ensayos realizados sobre los áridos extraídos de las viguetas de aluminoso, utilizadas en Cataluña, se considera éste insignificante; no obstante dicho aporte se comprueba periódicamente.
En los cálculos de la dosificación se considera como contenido medio de $\mathrm{Al}_{2} \mathrm{O}_{3}$ el $40 \%$.

$D=$ dosificación, en $\mathrm{kg}$ de cemento $/ \mathrm{m}^{3}$ de hormigón.

$A=\%$ (en peso) de $\mathrm{Al}_{2} \mathrm{O}_{3}$.

$\mathrm{d}=$ densidad, en $\mathrm{kg} / \mathrm{m}^{3}$.

\section{CONTENIDO EN AGUA}

El contenido en agua del hormigón se considera similar al contenido de agua combinada, que se toma por aproximación como la pérdida a fuego a $550^{\circ} \mathrm{C}$ del hormigón. Esta determinación no es todo lo exacta que sería de desear, pero se considera que dada la sencillez de su realización puede ser suficiente a efectos de comparar diversos hormigones entre sí. Se añade a ese resultado el valor de la porosidad (en \%), descontando el $2 \%$, por considerar esta proporción como correspondiente a aire ocluido.

Por consiguiente, el contenido en agua (A) se calcula por la fórmula siguiente:

$$
A=\operatorname{Pf}\left(550^{\circ}\right) \cdot d / 1.100+(p-2) 10
$$

donde:

Pf es la pérdida al fuego,

d es la densidad, en $\mathrm{kg} / \mathrm{m}^{3}, \mathrm{y}$

p es la porosidad, en \%.

\section{publicación del ICCET/CSIC}

\section{ACUEDUCTOS ROMANOS EN ESPAÑA Carlos Fernández Casado \\ Prof. Dr. Ing. de Caminos, Canales y Puertos}

Esta publicación se compone de una serie de artículos, publicados en la Revista "Informes de la Construcción", en los cuales se hace un análisis de los acueductos romanos que existen en España y el balance de las condiciones de conservación en que se encuentra cada uno de ellos, incluyendo referencias históricas y literarias. Se ha ilustrado con la reproducción de la valiosa documentación gráfica que posee el prestigioso autor.

Un volumen encuadernado en couché, a dos colores, de $21 \times 27$ centimetros, compuesto de 238 páginas, numerosos grabados, dibujos, fotos en blanco y negro y figuras de linea.

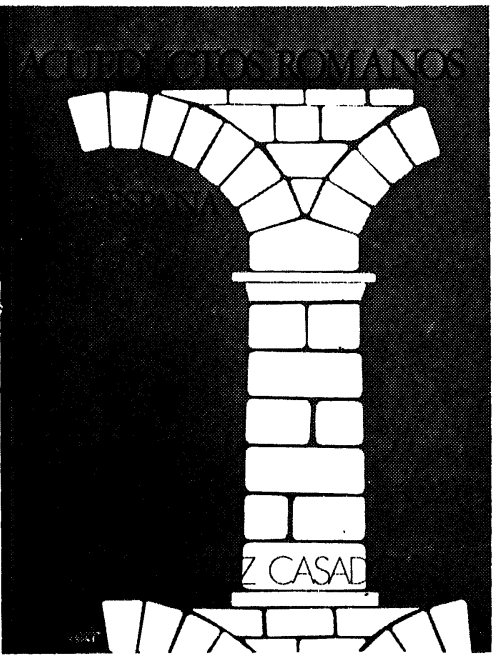

